\title{
Blockade of Th1 chemokine receptors ameliorates pulmonary granulomatosis
} in mice

\author{
J. Kishi*,+, Y. Nishioka*,+, T. Kuwahara*, S. Kakiuchi*, M. Azuma*, Y. Aono*, \\ H. Makino*, K. Kinoshita*, M. Kishi*, R. Batmunkh*, H. Uehara ", K. Izumi" and S. Sone*
}

ABSTRACT: Sarcoidosis is a granulomatous disease of unknown aetiology. We identified immunological targets for the treatment of pulmonary granulomatosis using a murine model generated with Propionibacterium acnes.

Sensitisation and challenge using heat-killed $P$. acnes and dendritic cells (DCs) were performed to produce pulmonary granulomatosis in C57BL/6 mice. Immunological analyses using ELISA as well as cDNA microarray analysis were used to search for cytokines or chemokines associated with the formation of granulomas in the lungs.

Co-administration of $P$. acnes and DCs reproducibly induced the formation of pulmonary granulomas, which resembled sarcoid granulomas. The cDNA microarray assay demonstrated that the gene expression of CXCL9 and CXCL10, ligands for CXCR3, and of CCL4, a ligand for CCR5, was strongly upregulated during granulomatosis. ELISA confirmed that levels of CXCL9 and CXCL10 as well as T-helper (Th)1 cytokines and chemokines including tumour necrosis factor- $\alpha$ and interferon- $\gamma$ were elevated in bronchoalveolar lavage fluid (BALF). The blockade of Th1 chemokine receptors using TAK-779, a dual blocker for CXCR3 and CCR5, led to reduced numbers of CXCR3+CD4+ and CCR5+CD4+ T-cells in BALF. Furthermore, administration of TAK779 ameliorated the granulomatosis.

The targeted inhibition of Th1 chemokines might be useful for inhibiting Th1-biased granulomatous diseases, including sarcoidosis.

KEYWORDS: CCR5, CXCR3, Propionibacterium acnes, pulmonary granulomatosis, Th1 chemokines

arcoidosis is a systemic granulomatous disease of unknown aetiology that affects mainly the lungs and lymphatic system [1-4]. Although spontaneous remission occurs in nearly two-thirds of cases, chronic and progressive courses are observed in $10-30 \%$ of patients [1-4]. Corticosteroids are used to treat sarcoidosis, but their effects are controversial, particularly in cases complicated with lung diseases [1-4]. In addition, long-term therapy with corticosteroids can result in serious adverse events [4]. Therefore, a new approach to the treatment of sarcoidosis with intensive organ involvement is needed.

The sarcoid granuloma is characterised by noncaseating epitheloid cells with the dominant accumulation of CD4+ T-cells and macrophages [1-4]. CD4+ T-lymphocytes are divided into two subgroups, $\mathrm{T}$ helper (Th) 1 and Th2 cells, on the basis of cytokine production [5]. Based on the Th1/ Th2 paradigm, sarcoidosis is considered a typical Th1-dominant disease, since T-lymphocytes in bronchoalveolar lavage fluid (BALF) and lymph nodes from patients with sarcoidosis predominantly produce interferon (IFN)- $\gamma$, interleukin (IL)- 2 and tumour necrosis factor (TNF) $-\alpha$ or $-\beta$ [6-8]. We and others have reported that levels of Th1 chemokines, including CXCL9/IFN- $\gamma$ (Mig), CXCL10/interferon- $\gamma$-inducible protein-10 (IP-10) and CXCL11/interferon-inducible T-cell $\alpha$-chemoattractant (I-TAC), were elevated in BALF of patients with sarcoidosis [9-13]. However, it is still not clear whether these Th1-related molecules would be useful targets in the treatment of pulmonary granulomatosis. The blockade of these molecules would probably benefit patients: in clinical

This article has supplementary material available from www.erj.ersjournals.com

AFFILIATIONS

Depts of *Respiratory Medicine and Rheumatology,

*Molecular Bacteriology, and

"Molecular and Environmental Pathology, Institute of Health Biosciences, The University of Tokushima Graduate School, Tokushima, Japan.

+These authors contributed equally to this work.

CORRESPONDENCE

Y. Nishioka

Dept of Respiratory Medicine and Rheumatology

Institute of Health Biosciences University of Tokushima Graduate School

3-18-15 Kuramoto-cho

Tokushima 770-8503

Japan

E-mail: yasuhiko@

clin.med.tokushima-u.ac.jp

Received:

May 052010

Accepted after revision:

Jan 062011

First published online:

Jan 272011 

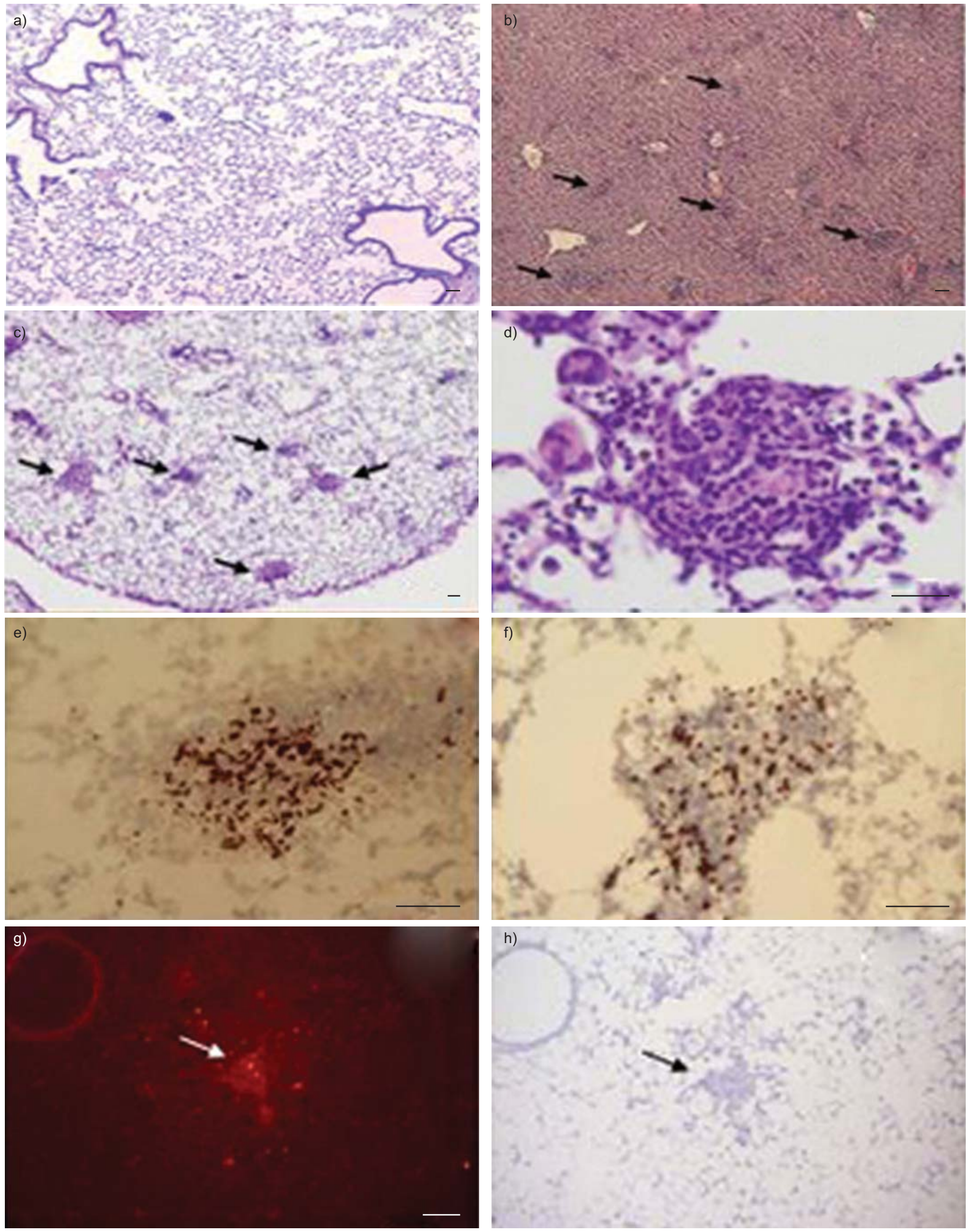

FIGURE 1. Figure legend is presented on the following page. 


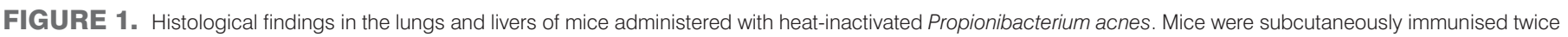

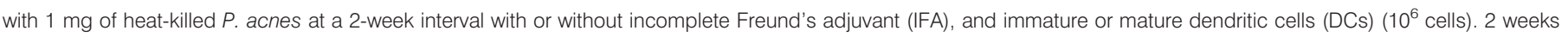

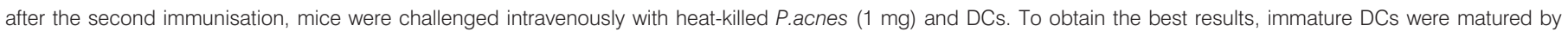

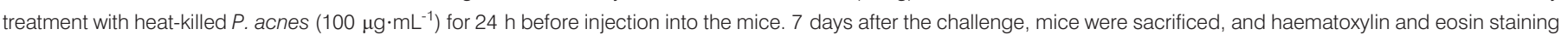

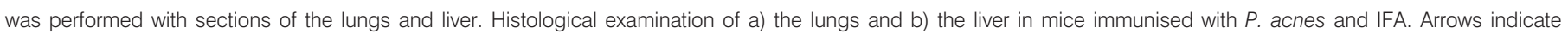

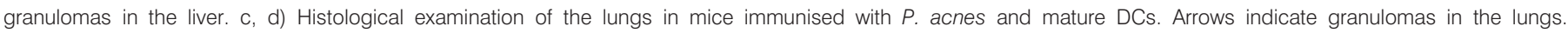

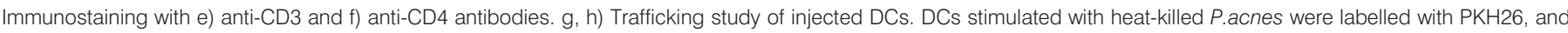

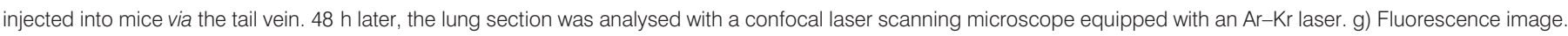

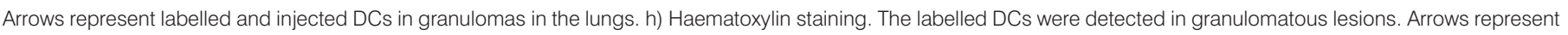
granulomas in the lungs. Scale bars $=100 \mu \mathrm{m}$.

studies with anti-TNF- $\alpha$ antibodies (infliximab and adalimumab), favourable results have been obtained in some cases [14, 15].

To identify novel molecular targets for the treatment of pulmonary granulomatosis, we report here the results of a global analysis of the mRNA expression of cytokines and chemokines using a cDNA microarray in a model of pulmonary granulomatosis generated by the systemic administration of Propionibacterium acnes and dendritic cells (DCs) in mice, because $P$. acnes has been reported to be involved in the aetiology of sarcoidosis [16-18].

\section{MATERIALS AND METHODS}

Detailed methods are described in the online supplementary material.

\section{Mice and materials}

8-week-old female C57BL / 6 mice were purchased from Charles River Japan Inc. (Yokohama, Japan), and maintained in the animal facility of the University of Tokushima (Tokushima, Japan) [19]. The small nonpeptide compound TAK-779 $(\mathrm{N}, \mathrm{N}-$ dimethyl- $N$-[4-[[[2-(4-methylphenyl)-6,7-dihydro-5H-benzocyclohepten-8-yl]carbonyl]amino]benzyl]tetrahydro-2H-pyran-4aminium chloride) was provided by Takeda Pharmaceutical Company (Osaka, Japan) [20]. TAK-779 is a selective antagonist of CXCR3 and CCR5 [21].

\section{P. acnes}

$P$. acnes was obtained from the American Type Culture Collection (Manassas, VA, USA) (ATCC \#6919) and grown in GAM broth (Nissui Pharmaceutical Co., Ltd, Tokyo, Japan). The suspension was heated by autoclaving, and was kept at $-70^{\circ} \mathrm{C}$ prior to use.

\section{Mouse bone marrow-derived DCs}

The bone marrow (BM)-derived DCs were generated using recombinant mouse granulocyte-macrophage-colony stimulating factor (Kirin Brewer Inc., Tokyo, Japan) and recombinant mouse IL-4 (Pepro Tech, London, UK) [22].

\section{Sensitisation and challenge of mice with heat-killed P. acnes}

Mice were subcutaneously immunised twice with heat-killed $P$. acnes with or without incomplete Freund's adjuvant (IFA) or DCs. Mice were then challenged intravenously with heat-killed $P$. acnes with or without DCs.

\section{Bronchoalveolar lavage}

Bronchoalveolar lavage was performed as described previously [23].

\section{Measurements of CXCR3 ligands and Th1 and Th2 cytokines}

Levels of CXCL9 and CXCL10 were examined by ELISA (R\&D System, Minneapolis, MN, USA) [23]. Th1 and Th2 cytokines were measured using the BD Cytometric Beads Array System mouse Th1/Th2 cytokine kit (Becton Dickinson Company, Franklin Lakes, NJ, USA).

\section{Trafficking study of injected DCs}

DCs $\left(10^{6}\right.$ cells) were stained with PKH26 (Sigma, St. Louis, $\mathrm{MO}, \mathrm{USA})$, and injected into the tail vein. Fluorescence images of sections excited at $568 \mathrm{~nm}$ were captured with a confocal laser scanning microscope (Leica TCS NT; Leica, Heidelberg,

\section{TABLE 1 Pulmonary granulomatosis in mice immunised with heat-killed Propionibacterium acnes and dendritic cells (DCs)}

\begin{tabular}{lccc} 
Method of immunisation & Experiment & Granulomas & Size $\boldsymbol{\mu m}^{\mathbf{2}}$ \\
\hline P. acnes + IFA & & & \\
& 2 & 5 & $2880 \pm 1731$ \\
& 3 & 4 & $2137 \pm 1132$ \\
& Total & $3.6 \pm 1.5$ & $2413 \pm 1426$ \\
P. acnes + immature DCs & & & \\
& 1 & 22 & $5195 \pm 4172$ \\
& 2 & 9 & $2025 \pm 1153$ \\
& 3 & 8 & $3318 \pm 2054$ \\
Mature DCs pulsed with P. acnes & Total & $13 \pm 7.8$ & $4132 \pm 3933$ \\
& 1 & & \\
& 2 & 32 & $7980 \pm 8941$ \\
& 3 & 21 & $7682 \pm 6999$ \\
& Total & $24.7 \pm 6.3^{*}$ & $7960 \pm 7311^{*}$
\end{tabular}

Data are presented as $n$ or mean \pm SD. Mice were subcutaneously immunized twice with $1 \mathrm{mg}$ of heat-killed Propionibacterium acnes at a 2-week interval. 2 weeks after the second immunisation, mice were challenged intravenously with heat-killed $P$. acnes $(1 \mathrm{mg})$. The heat-killed $P$. acnes was administered with or without incomplete Freund's adjuvant (IFA), immature or mature DCs $\left(10^{6}\right.$ cells). Mature DCs were generated by pulsing with heat-killed $P$. acnes $\left(100 \mu \mathrm{g} \cdot \mathrm{mL}^{-1}\right)$ for $24 \mathrm{~h}$ before their injection into mice. 7 days after the challenge, mice were sacrificed, and haematoxylin and eosin staining was performed on sections of the lungs. The granulomatosis was evaluated by measuring the number and size of granulomas in the lungs. Similar results were obtained in three separate experiments. ${ }^{*}: \mathrm{p}<0.05$ versus $P$. acnes plus IFA group. 
Germany) equipped with an Ar-Kr laser and a $\times 10$ dry objective (Leica Plan Apochromat).

\section{Isolation of total RNA}

Total RNA was isolated from the lungs of mice using Isogen (Wako K.K., Kyoto, Japan) [24].

\section{DNA microarrays}

A description of mouse cytokine and chemokine microarrays that contained probes for the genes of 29 cytokines, 34 cytokine receptors, 33 chemokines and 21 chemokine receptors (Kakengeneqs Co. Ltd, Chiba, Japan) is given in online supplementary table E1 [25].

\section{cDNA preparation and array hybridisation}

Total RNA samples $(100 \mu \mathrm{g})$ were converted to doublestranded cDNA using a custom kit (LavelStar Array kit; Qiagen, Valencia, CA, USA) and labelled with cyanine 3conjugated deoxyuridine triphosphate (dUTP) [25]. Reference total RNA was labelled with cyanine 5-conjugated dUTP (PerkinElmer, Boston, MA, USA). Array hybridisation was performed according to the manufacturer's instructions [25].

\section{Microarray quantification}

Fluorescence images of hybridised microarrays were obtained using an array scanner (model 428; Affymetrix, Santa Clara, CA, USA) [25]. Raw fluorescence intensity data were used to calculate signal intensities of the spots (DNASIS Array; Hitachi
Software Engineering Co., Ltd, Tokyo, Japan). A two-fold change in gene expression was used as the cut-off.

\section{Histopathology}

Left lungs were fixed in buffered $10 \%$ formalin and embedded in paraffin. Sections were stained with haematoxylin and eosin [23].

\section{Immunohistochemistry}

$6-\mu \mathrm{m}$ sections of the left lungs were stained using the R.T.U. VECTASTAIN Universal Quick Kit (Vector Laboratories, Burlingame, CA, USA) [25].

\section{Administration of TAK-779}

TAK-779 was dissolved in 5\% mannitol solution, and was administered subcutaneously into the mice.

\section{Flow cytometry and immunofluorescence staining}

Flow cytometric analysis was performed using FACSCalibur (BD Biosciences, Franklin Lakes, NJ, USA) [19, 22]. Immunofluorescence staining was visualised using a fluorescence microscope (Olympus BX61; Olympus Optical Co. Ltd, Tokyo, Japan).

\section{Statistical analysis}

Comparisons among multiple groups were performed using one-way ANOVA with Newman-Keuls post hoc correction (GraphPad Prism, version 3.0; GraphPad Software, La Jolla, CA, USA). Values of $\mathrm{p}<0.05$ were considered statistically significant. Data are presented as mean \pm SD.
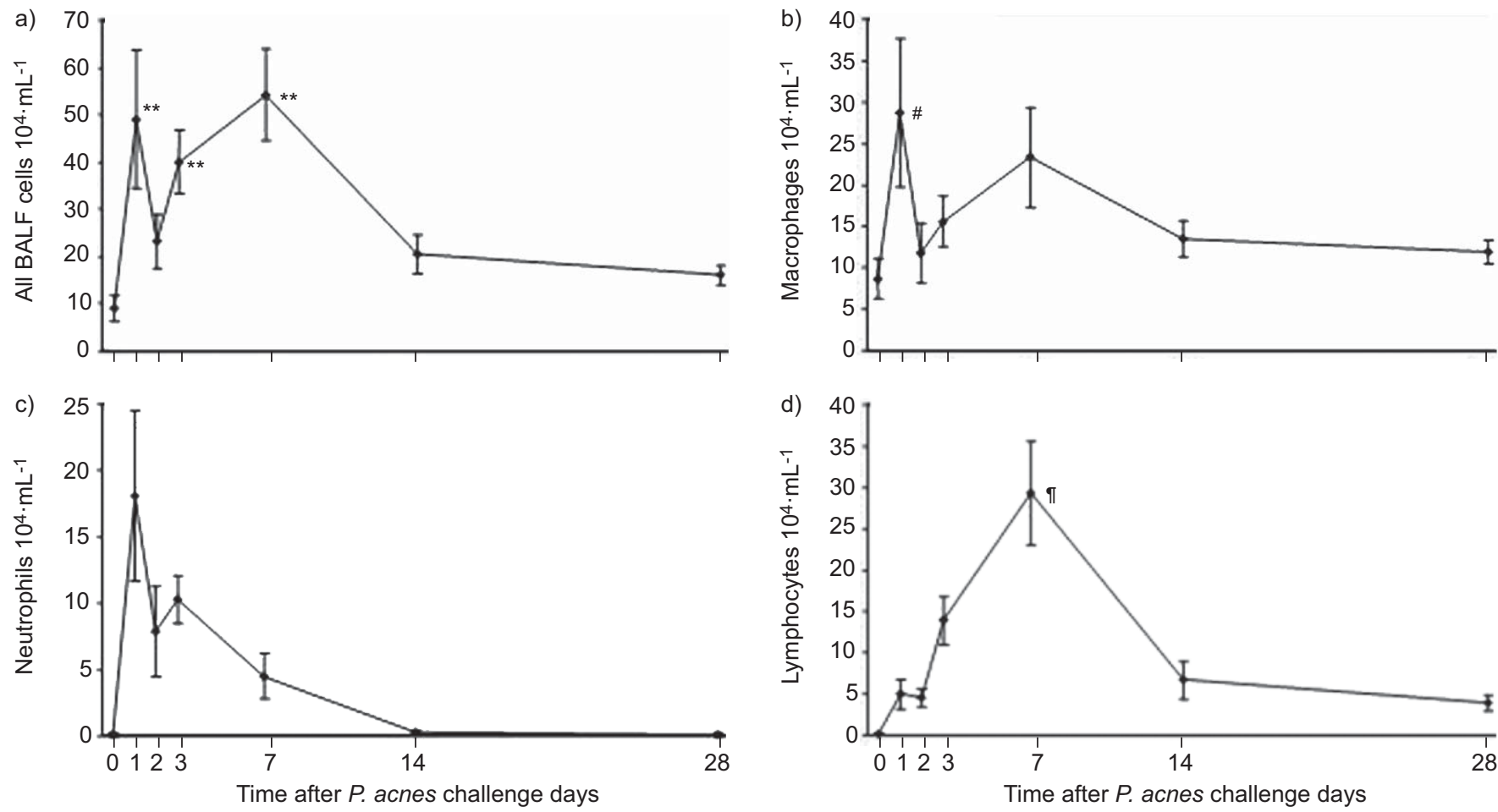

FIGURE 2. Analysis of bronchoalveolar lavage fluid (BALF) of mice with pulmonary granulomatosis induced by Propionibacterium acnes and dendritic cells (DCs). Mice were subcutaneously immunised twice with $1 \mathrm{mg}$ of heat-killed $P$. acnes and mature DCs at a 2-week interval. 2 weeks after the second immunisation, mice were challenged intravenously with heat-killed $P$. acnes $(1 \mathrm{mg})$ and mature DCs $\left(10^{6}\right.$ cells). On days $0,1,2,3,7,14$ and 28 after the challenge, bronchoalveolar lavage was performed. Data are presented as mean $\pm S D$ for the group of four mice. Similar results were obtained in three separate experiments. ${ }^{* *}: p<0.001$ versus day $0 ;{ }^{*}: p=0.02 ;{ }^{\top}: p=0.01$. 


\section{RESULTS}

\section{Administration of heat-inactivated P. acnes with DCs} enhanced the formation of pulmonary granulomas in mice We first examined whether immunisation with heat-inactivated $P$. acnes could induce granulomas to form in mouse lungs. C57BL/6 mice were subcutaneously sensitised twice with $P$. acnes and IFA at a 2-week interval. 2 weeks after the second sensitisation, mice were challenged with an i.v. injection of heat-killed $P$. acnes. As reported previously [26], many granulomatous lesions were developed in the liver, but not in the lungs, after immunisation with heatinactivated $P$. acnes and IFA (fig. $1 \mathrm{a}$ and $\mathrm{b}$ ). When BM-DCs were co-administered with $P$. acnes in the immunisation as well as the challenge process, the number and size of pulmonary granulomas were significantly increased (table 1; fig. 1c and d). These granulomas consisted of lymphocytes, epithelioid cells, and multinucleated giant cells (fig. 1d; online supplementary figs E1 and E2), quite similar to those observed in the lungs of patients with sarcoidosis.
Granuloma size was largest on day 7, and gradually reduced until day 14 (online supplementary fig. E2). Immunohistochemical analysis showed CD3+ and CD4+ lymphocytes in the pulmonary granulomas (fig. $1 \mathrm{e}$ and $\mathrm{f}$ ). The trafficking study of labelled DCs showed that the injected DCs were distributed into the lungs $6 \mathrm{~h}$ after injection, and the granuloma appeared $24 \mathrm{~h}$ later. In the granulomatous lesion, some labelled DCs were detected until $72 \mathrm{~h}$ after injection. These data demonstrated that the injected DCs migrated into the lungs via blood circulation and induced an immune reaction to generate the granulomatous lesions in situ (online supplementary fig. E5).

\section{Analysis of BALF of mice with pulmonary granulomatosis induced by $P$. acnes}

To clarify which type of cell is involved in the granulomatosis caused by P. acnes, we analysed cells in the BALF of mice. As shown in figure 2, neutrophils in BALF increased in number rapidly 1 day after the challenge with $P$. acnes, but had
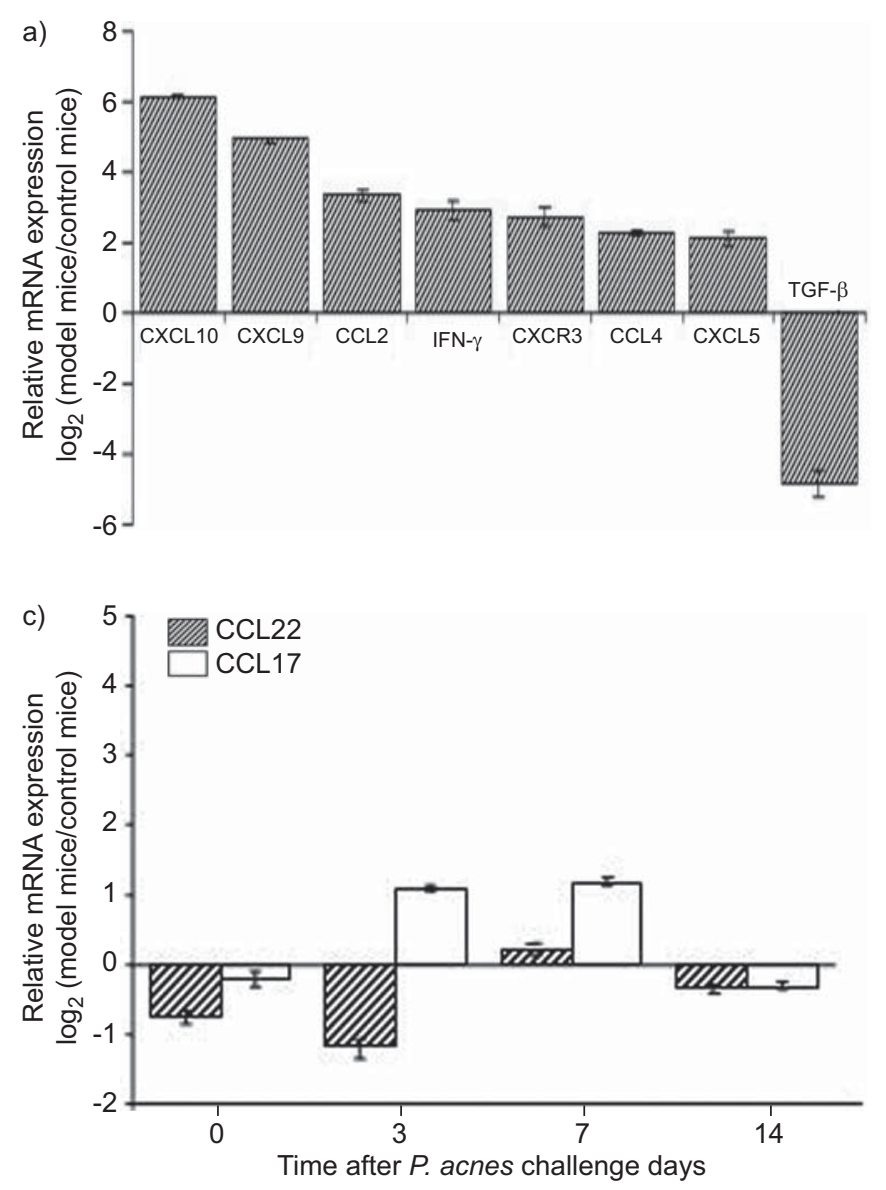

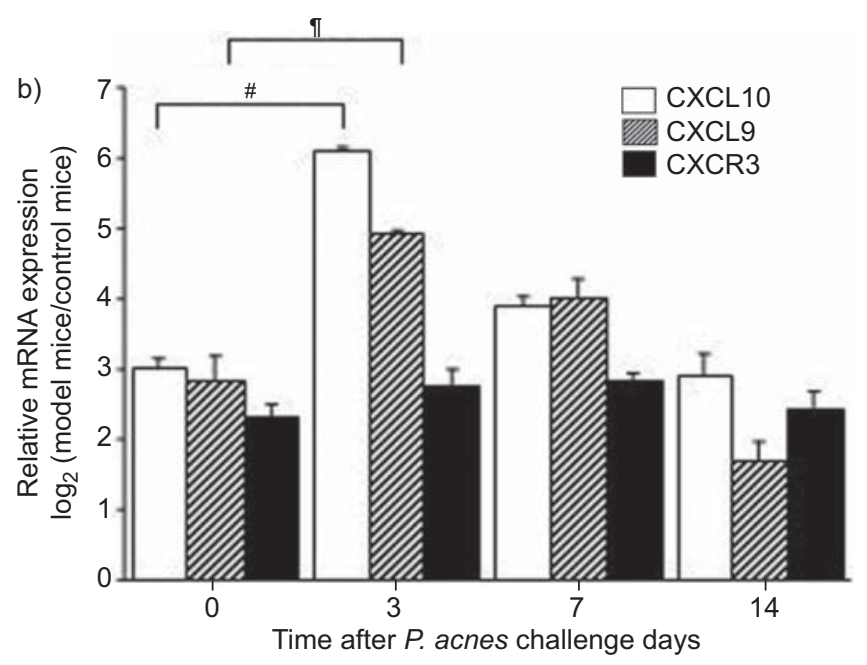

FIGURE 3. Change in gene expression in mice with pulmonary granulomatosis induced by Propionibacterium acnes and dendritic cells (DCs). Total RNA was isolated from the lungs of untreated mice as well as $P$. acnes-immunised mice before or 3,7 or 14 days after $i . v$. administration of $P$. acnes with DCs. Briefly, the lungs were quickly perfused with $5 \mathrm{~mL}$ of cold PBS through the right ventriculum. The lungs were then homogenised in $1 \mathrm{~mL}$ of Isogen (Wako K.K., Kyoto, Japan), total RNA was extracted and a cDNA microarray analysis was performed as described in the online supplement. Data are presented as mean \pm SD. a) Major upregulated genes in the lungs of mice 3 days after $P$. acnes challenge. b) Time course of the gene expression of CXCL9, CXCL10 and CXCR3 in the lungs of mice after $P$. acnes challenge. c) Time course of the gene expression of CCL17 and CCL22 in the lungs of mice after the $P$. acnes challenge. IFN: interferon; TGF: transforming growth factor. ${ }^{\#}: p=0.007 ;{ }^{\bullet}: p=0.008$. 
disappeared by day 14 . However, the number of lymphocytes rose gradually and reached a peak 7 days after the challenge, then decreased until day 28 . Two peaks in the number of macrophages were found, 1 and 7 days after the $P$. acnes challenge, with a gradual decline from day 7 to day 28. These changes were correlated with the histological findings (data not shown).

\section{Levels of Th1 chemokines were elevated during pulmonary granulomatosis induced by $\mathbf{P}$. acnes}

Next, we analysed which cytokines and chemokines are involved in the pulmonary granulomatosis induced by $P$. acnes, using a cDNA microarray focusing on cytokines and chemokines (online supplementary table E1). As shown in figure 3a, mRNA expression of the Th1 chemokines CXCL9 and CXCL10 was strongly stimulated. mRNAs of other Th1related molecules including CCL4, CXCL5, CXCR3 and IFN- $\gamma$ were also expressed strongly after $P$. acnes challenge. The mRNA of CCL2 also increased in the granulomatous lungs, consistent with reports describing pulmonary granulomas induced by $P$. acnes in rabbits [27]. The increase in CXCL9 and CXCL10 occurred 3 to 7 days after the challenge with $P$. acnes (fig. $3 b$ ). However, levels of the Th2 chemokines CCL17 and CCL22 did not increase during the course of the pulmonary granulomatosis (fig. 3c).

To confirm the stimulation of Th1 chemokines and cytokines, we performed an ELISA with BALF. As shown in figure 4, CXCL9, CXCL10 and IFN- $\gamma$ levels were markedly elevated after challenge with $P$. acnes. TNF- $\alpha$ also increased in BALF, but the difference did not reach statistical significance. However, Th2 cytokines, including IL-4 and IL-5, were not detected in the course of pulmonary granulomatosis (data not shown).

\section{Therapy targeting Th1 chemokines ameliorates the pulmonary granulomatosis caused by $\mathbf{P}$. acnes}

As we confirmed the elevation in levels of the Th1 chemokines CXCL9, CXCL10 and CCL4 in this model, we hypothesised that the blockade of Th1 chemokines might be effective in inhibiting pulmonary granulomatosis. To test this, we used TAK-779, a selective blocker of the Th1 chemokine receptors CXCR3 and CCR5.
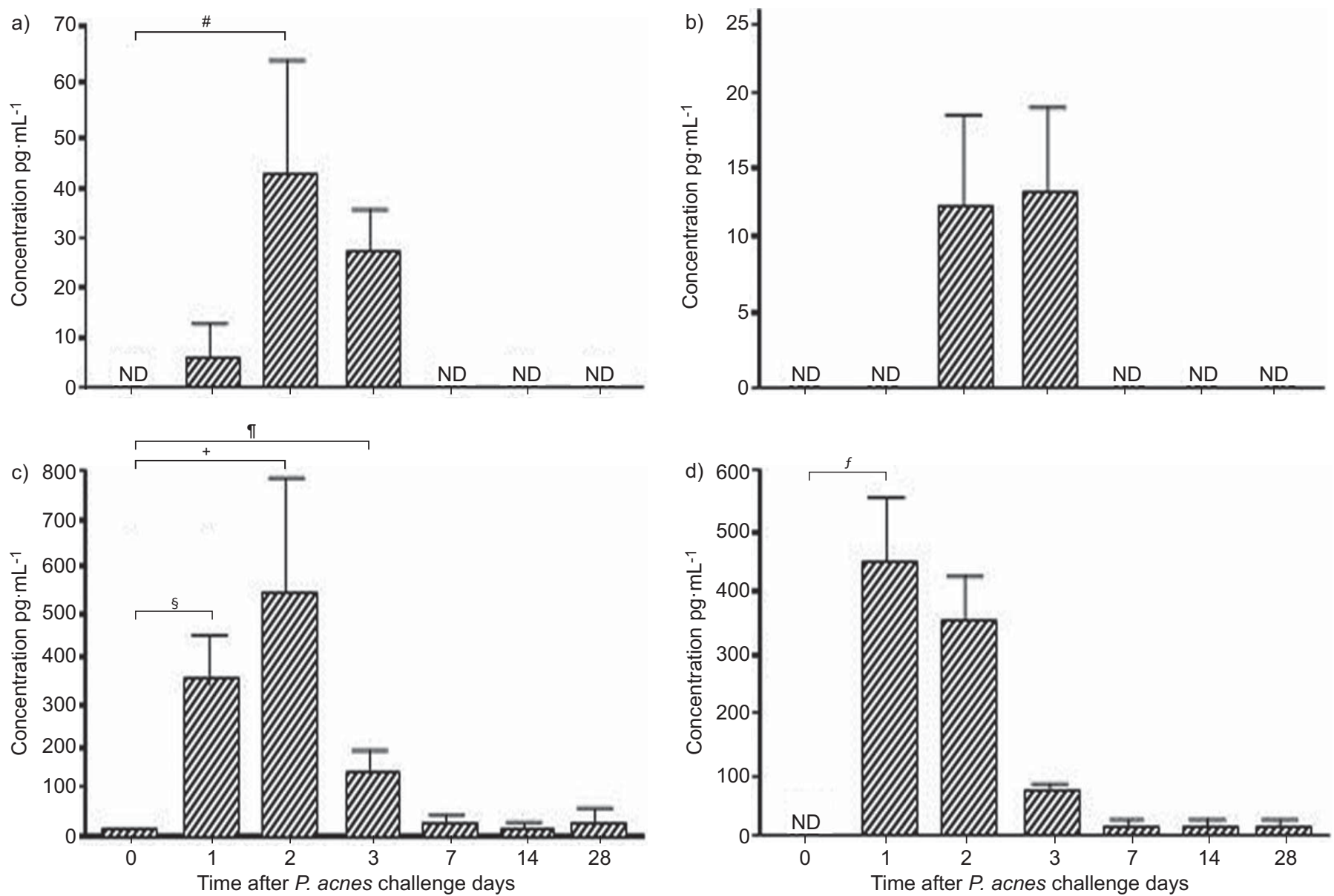

FIGURE 4. Elevation in levels of Thelper (Th) 1 cytokines and chemokines in bronchoalveloar lavage fluid (BALF) of mice with pulmonary granulomatosis generated by Propionibacterium acnes. Mice were anaesthetised and a soft cannula (23G) was inserted into the trachea. Bronchoalveolar lavage was performed five times with the instillation and withdrawal of $1 \mathrm{~mL}$ of saline at various time points. BALF was stored at $-80^{\circ} \mathrm{C}$ until used. The frozen BALF was quickly thawed and used to examine concentrations of cytokines and chemokines. a) Th1 (interleukin (IL)-2, IL-4, IL- 5 and interferon- $\gamma$ ) and b) Th2 (tumour necrosis factor- $\alpha$ ) cytokines were measured using the BD Cytometric Beads Array System. Mouse c) CXCL9 and d) CXCL10 levels were measured by ELISA. Data are presented as mean \pm SD for the group of four mice. Similar results were obtained in three separate experiments. ${ }^{\#}: p=0.034 ;{ }^{\circ}: p=0.025 ;{ }^{+}: p=0.009 ;{ }^{s}: p=0.018 ;{ }^{f}: p=0.05$. 

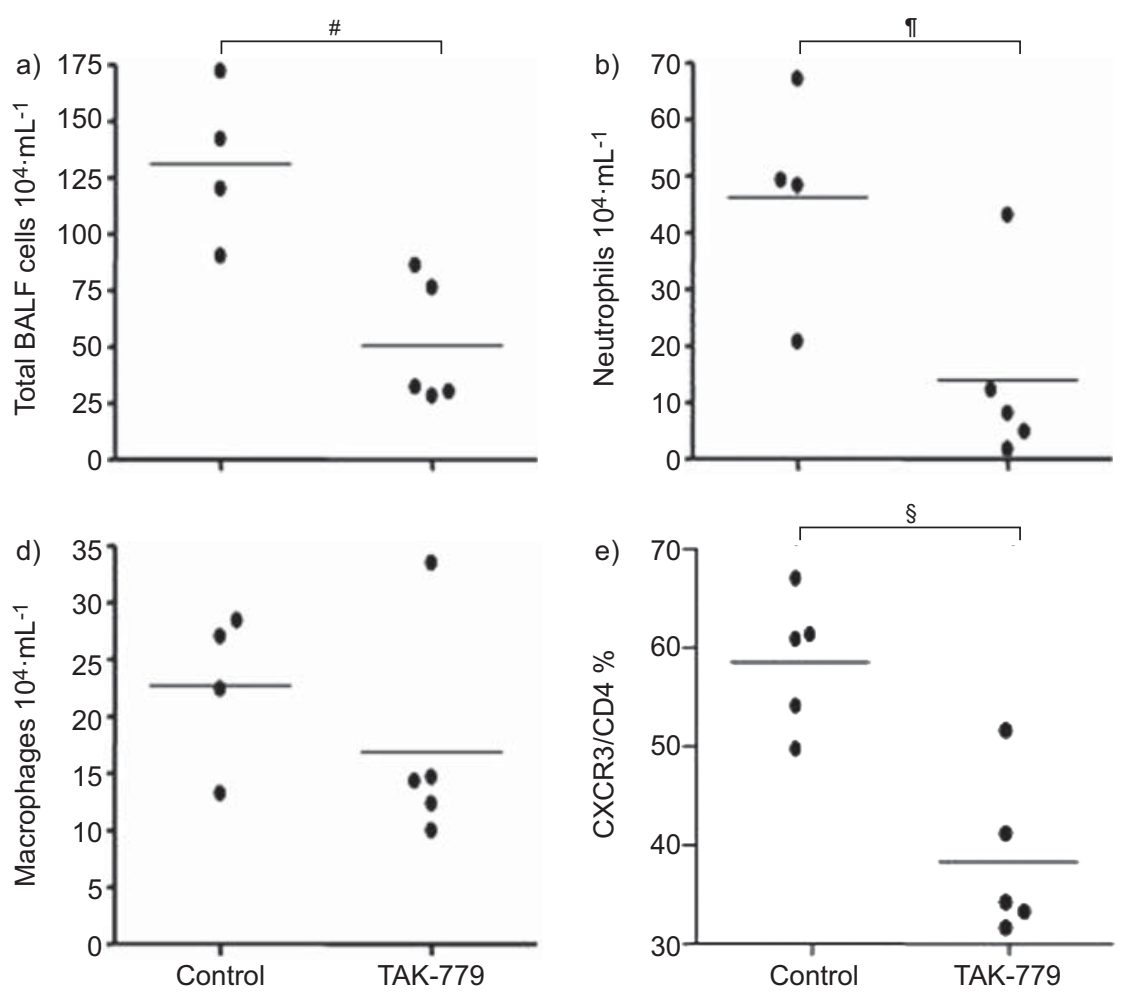
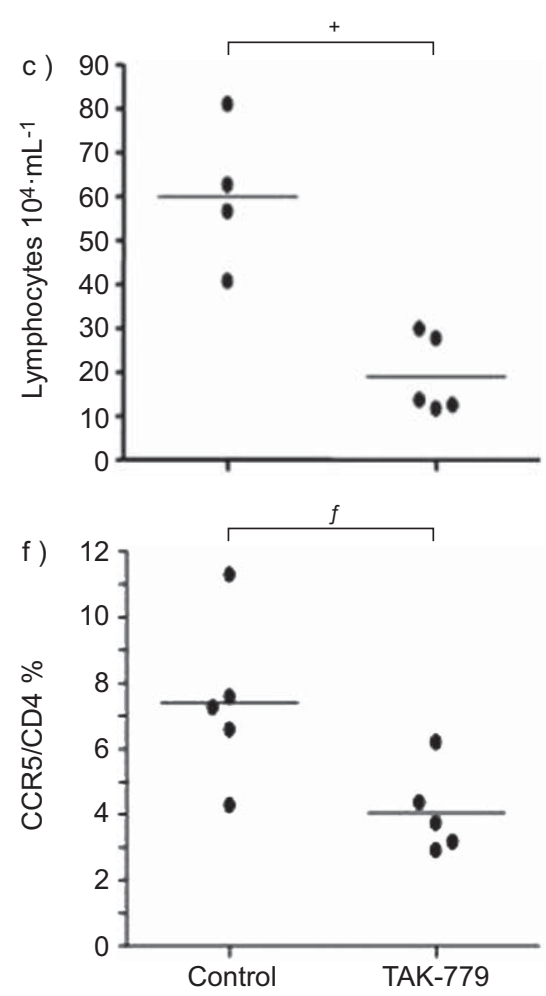

FIGURE 5. Administration of TAK-779 blocks the trafficking of T helper 1 lymphocytes into the lungs. TAK-779 (150 $\mu$ g.day $\left.{ }^{-1}\right)$ was administered subcutaneously from 4 days before to 6 days after challenge with heat-inactivated Propionibacterium acnes and mature dendritic cells every other day. Bronchoalveolar lavage (BAL) analysis was performed 7 days after the challenge. Mice were anaesthetised and a soft cannula (23G) was inserted into the trachea. BAL was performed five times with the instillation and withdrawal of $1 \mathrm{~mL}$ of saline at various time points. The total cell count of the BAL fluid (BALF) was determined using Turk staining solution. BALF was centrifuged, and the cell pellets were resuspended into saline and cytospun onto glass slides. These cells were stained with Diff-Quick staining solution (Baxter, Miami, FL, USA), and 200 cells were counted for cell classification. The numbers of a) all cells, b) neutrophils, c) lymphocytes and d) macrophages in BALF of mice treated with control (5\% mannitol solution) or TAK-779. Data are presented as mean \pm SD for each group of four or five mice. The percentages of e) CXCR3+CD4+ and f) CCR5+CD4+ T-cells in BALF of mice treated with control (5\% mannitol solution) or TAK-779. BAL cells were analysed by flow cytometry. CXCR3+CD4+ and CCR5+CD4+ T-cells were determined with two-colour fluorescence staining. Data are presented as the mean \pm SD for the group of five mice. Data are representative of three separate experiments. ${ }^{\#}: p=0.011 ;{ }^{\top}: p=0.015 ;{ }^{+}: p=0.005$; $s: p=0.016 ;{ }^{f}: p=0.02$.

Mice were treated every other day with TAK-779 from 4 days before to 6 days after the challenge with heat-inactivated $P$. acnes and DCs. TAK-779 significantly reduced the total number of cells as well as numbers of both neutrophils and lymphocytes (fig. 5a-d). The proportions of CXCR3+CD4+ T-cells and CCR5+CD4+ T-cells in BALF determined using flow cytometry were $50-68 \%$ and $4-12 \%$, respectively, indicating Th1-skewed immune responses in this model (fig. 5e and f). Treatment with TAK-779 clearly reduced the numbers of both CXCR3+CD4+ and CCR5+CD4+ T-cells (control versus TAK-779: CXCR3+CD4+; $58.54 \pm 6.75 \%$ versus $38.29 \pm 8.24 \%, p=0.016$; CCR $5+C D 4+; 7.37 \pm 2.52 \%$ versus $4.05 \pm 1.31 \%, p=0.020$ ). Furthermore, histological examination confirmed major reductions in both the number and size of pulmonary granulomas generated by $P$. acnes following the administration of TAK779 (control versus TAK-779: size; 7,033.14 $\pm 1,889.6$ versus $4,233.27 \pm 576.57 \mu^{2}, \quad p=0.008$; number; $25.4 \pm 5.89$ versus $15.2 \pm 4.20, \mathrm{p}=0.032)$ (fig. 6).

\section{DISCUSSION}

In the present study, we generated a murine model of pulmonary granulomatosis by co-administering $P$. acnes and DCs. Examination of the expressions of cytokines and chemokines in the lungs demonstrated that levels of Th1-type cytokines and chemokines were strongly elevated in this model. In addition, the Th1 chemokine receptor blocker TAK-779 inhibited the formation of granulomas in the lungs by reducing the numbers of CXCR3+CD4+ and CCR5+CD4+ T-cells accumulated in the lungs.

In this study, mice were sensitised twice with systemic administrations of heat-inactivated $P$. acnes and BM-derived DCs to generate pulmonary granulomas. A series of microbiological and molecular investigations have suggested that Propionibacteria spp. are aetiologically linked to sarcoidosis [16-18]. Using heat-inactivated P. acnes, several models of pulmonary granulomatosis have been established [26, 28]. MCCASKILL et al. [28] showed that the intratracheal administration of heat-killed $P$. acnes induced granulomatosis in the lungs of mice. Since it is likely that the intratracheal administration of heat-killed $P$. acnes induces nonspecific inflammation in the lungs, we selected systemic administration as it is more relevant to the condition in humans. We found that the challenge with $P$. acnes-pulsed DCs, which have the phenotype of mature DCs (online supplementary fig. E3), without heat-inactivated $P$. acnes also could induce many large 
a)
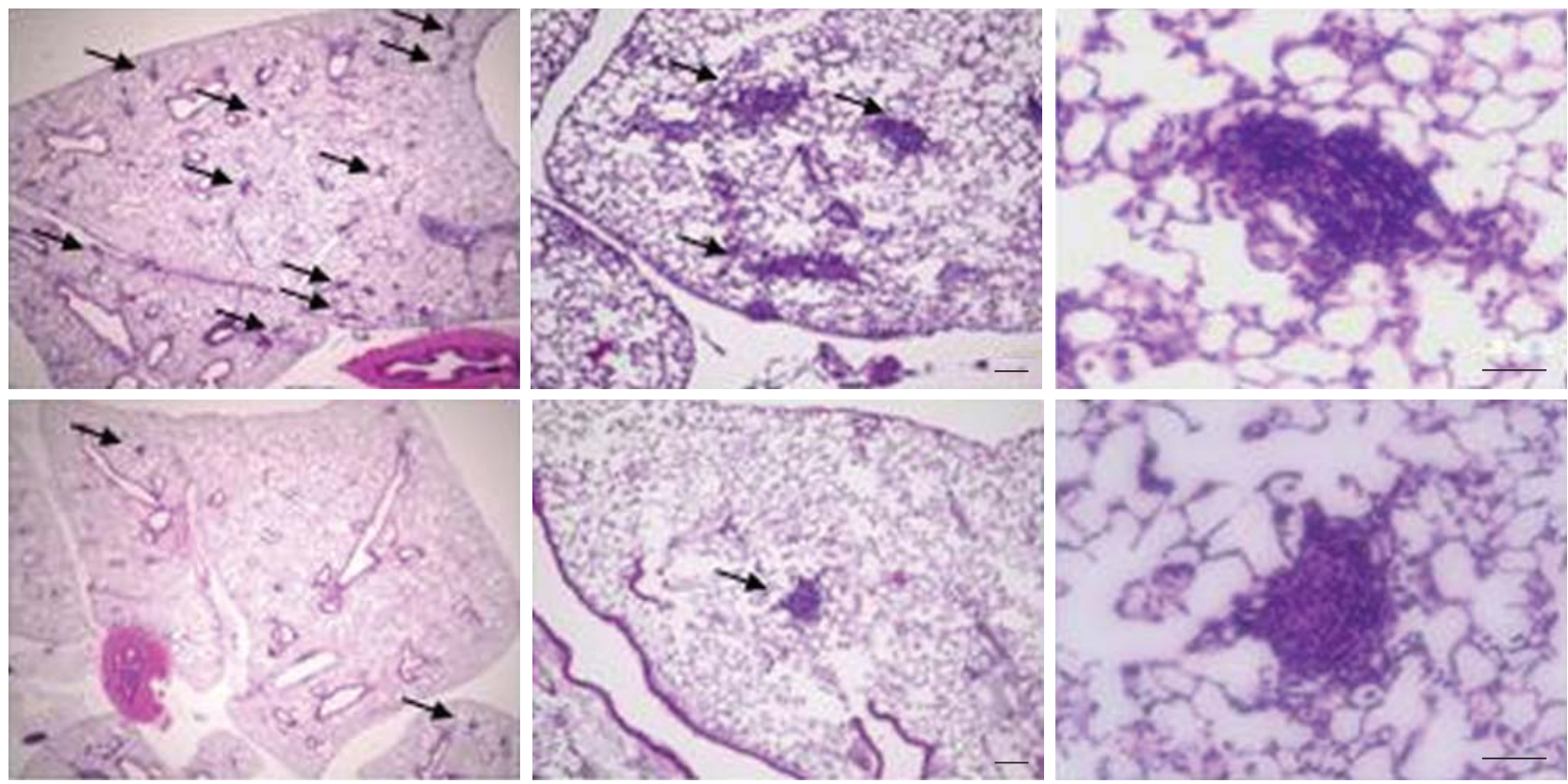

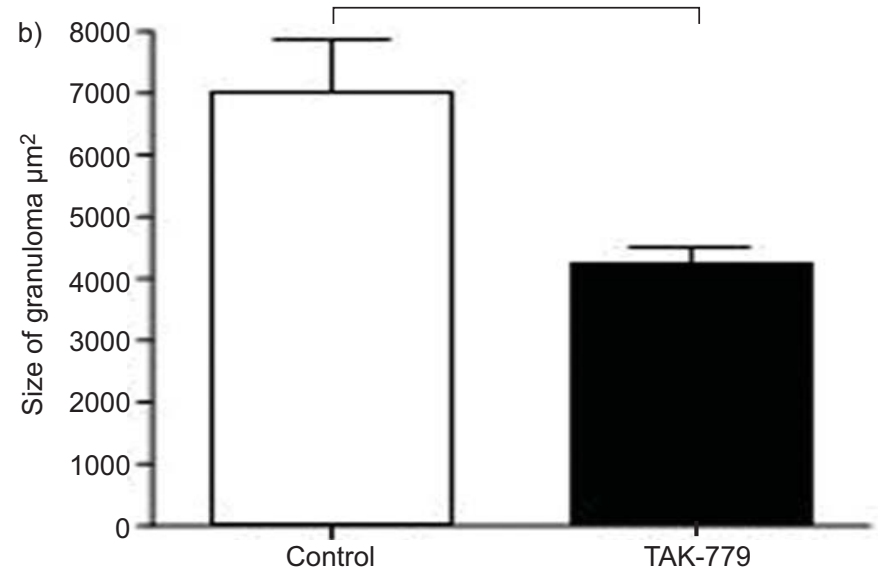

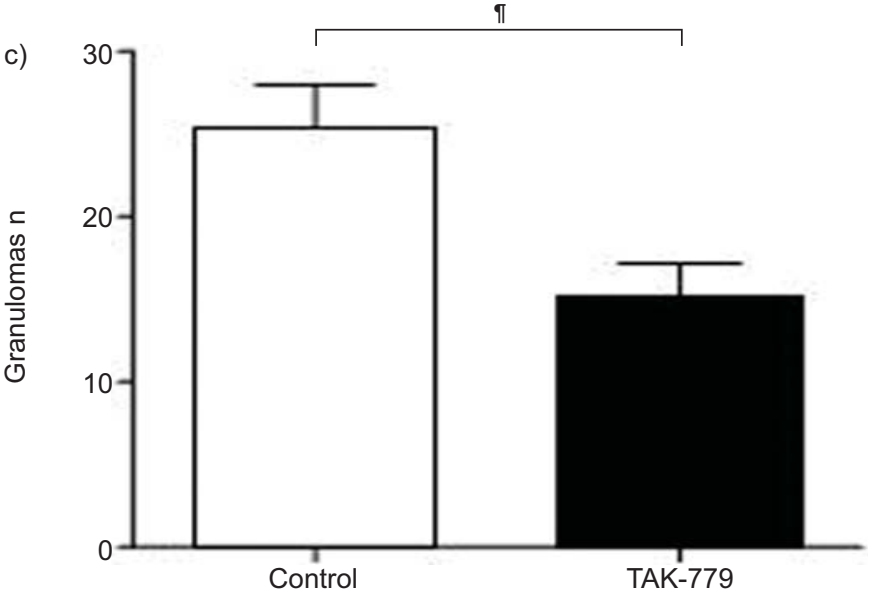

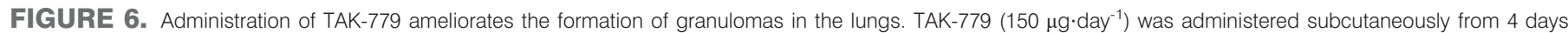

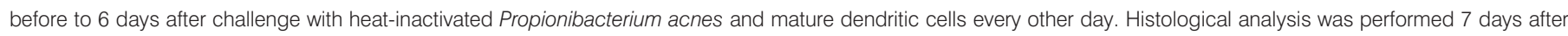

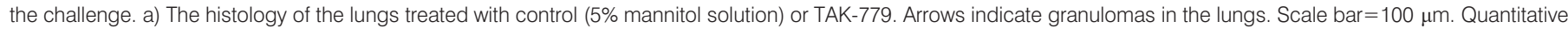

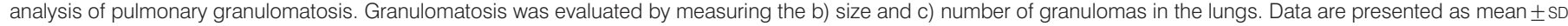
of five mice in the group. Similar results were obtained in three separate experiments. ${ }^{*}: p=0.008 ;{ }^{\bullet}: p=0.032$

granulomas in the lungs, indicating that the pulmonary granulomatosis seen in this model was mediated by immune reactions against $P$. acnes. NisHIWAKI et al. [26] also reported that repeated immunisation with heat-killed $P$. acnes using complete Freund's adjuvant (CFA) induced pulmonary granulomatosis [26], although we could not reproducibly generate granulomas using similar methods with IFA. The reason for this discrepancy is not clear, but it is likely to be due to the difference in the route and dose of immunisation with heatkilled $P$. acnes or adjuvant. In particular, CFA may not be suitable for inducing specific immune responses to $P$. acnes, since it contains derivatives of Mycobacterium tuberculosis. The granulomas found in previous reports consisted of lymphocytes, macrophages and epithelioid cells without multinucleated cells. However, the granulomas observed in our study contained multinucleated giant cells, being most similar to those in cases of human sarcoidosis. The DCs used in the immunisation might play a critical role because DCs were reported to be key immune cells in initiating granulomatous cell-mediated immunity [29].

In previous reports, levels of Th1 cytokines including IFN- $\gamma$, TNF- $\alpha$ and IL-12 were elevated in models of pulmonary granulomatosis induced by $P$. acnes [26, 29], whereas there is no published report analysing chemokines. Here, we showed significant upregulation of CXCL9 and CXCL10 expression during pulmonary granulomatosis induced by heat-killed $P$. acnes. Furthermore, these changes were strongest among cytokines and chemokines tested in the cDNA microarray system. This suggests these chemokines are ideal molecular 
targets for regulating Th1-type granulomas in the lungs, because they play critical roles in the trafficking of immune cells, particularly Th1 cells, into the lungs. In our model, CXCR3+CD4+ T-cells probably play more of a role than CCR5+CD4+ T-cells since the proportions of these cells were $50-68 \%$ and $4-12 \%$, respectively, which is consistent with an analysis in patients with sarcoidosis [30].

TAK-779 has a unique profile, selectively blocking both CXCR3 and CCR5 $[19,20]$. To test whether the blockade of Th1 chemokines attenuates pulmonary granulomatosis caused by $P$. acnes, we treated the sensitised mice with TAK-779 during the challenge with $P$. acnes. Administration of TAK-779 strongly inhibited pulmonary granulomatosis by preventing the accumulation of CXCR3+CD4+ and CCR5+CD4+ T-cells into the lungs. There are two ways to inhibit the interaction between a ligand and a receptor: neutralise the ligand or block the receptor. With chemokines, unlike cytokines, many ligands can bind a single receptor. Consequently, blocking the receptor would be more effective than blocking the ligands. Furthermore, TAK-779 has the advantage of blocking both Th1 chemokine receptors. This characteristic of TAK-779 might lead to the significant reduction in the accumulation of Th1 cells, and inhibit granulomatosis in the lungs. In turn, these observations also indicate that Th1 chemokines are crucial regulators in the formation of pulmonary granulomas by P. acnes. Furthermore, we found a reduced number of neutrophils in the BALF of TAK779-treated mice. This is not surprising: it has been reported that the neutrophils can also express the Th1 chemokine receptors CXCR3 and CCR5 [31, 32]. In addition, we could not rule out the possibility that the inhibitory effects of TAK-779 were partly mediated by blocking the influx of injected DCs into the lungs, since $P$. acnes-stimulated DCs also express CCR5 and CXCR3 (online supplementary fig. E4). Unlike human sarcoidosis, the condition in our model is not chronic and improved within 2 weeks, which is consistent with the previous reports, but is a limitation of our study [26, 28]. Recently, SAMOKHIN et al. [33] reported a model of prolonged pulmonary granulomatosis like human sarcoidosis in ApoE -/- mice. Further study is required to confirm the efficacy of TAK-779 in chronic models with therapeutic protocols.

In summary, we have demonstrated that production of Th1 chemokines is strongly stimulated during pulmonary granulomatosis caused by P. acnes with DCs, and targeted inhibition of Th1 chemokines with receptor blockers might be useful for inhibiting Th1-biased granulomatous diseases including sarcoidosis.

\section{SUPPORT STATEMENT}

This work was supported by KAKENHI (18590855 and 20390231), a Grant-in-Aid for Scientific Research, categories (C) and (B) from the Ministry of Education, Culture, Sports, Science and Technology (MEXT), and a grant to the Diffuse Lung Diseases Research Group from the Ministry of Health, Labour and Welfare, Japan.

\section{STATEMENT OF INTEREST}

None declared.

\section{ACKNOWLEDGEMENTS}

The authors thank T. Oka (Dept of Respiratory Medicine and Rheumatology, Institute of Health Bioscience, University of Tokushima, Tokushima, Japan) for technical assistance.

\section{REFERENCES}

1 American Thoracic Society, European Respiratory Society, World Association of Sarcoidosis and Other Granulomatous Disorders. Statement on sarcoidosis. Am J Respir Crit Care Med 1999; 160: 736-755.

2 Iannuzzi MC, Rybicki BA, Teirstein AS. Sarcoidosis. N Engl J Med 2007; 357: 2153-2165.

3 Paramothayan S, Lasserson T. Treatment for pulmonary sarcoidosis. Respir Med 2008; 102: 1-9.

4 Nunes H, Soler P, Valeyre D. Pulmonary sarcoidosis. Allergy 2005; 60: $565-582$.

5 Bonecchi R, Bianchi G, Bordignon PP, et al. Differential expression of chemokine receptors and chemotactic responsiveness of type 1 T helper cells (Th1s) and Th2s. J Exp Med 1998; 187: 129-134.

6 Wahlstrom J, Katchar K, Wigzell H, et al. Analysis of intracellular cytokines in $\mathrm{CD}^{+}$and $\mathrm{CD} 8^{+}$lung and blood T cells in sarcoidosis. Am J Respir Crit Care Med 2001; 163: 115-121.

7 Prasse A, Georges CG, Biller H, et al. Th1 cytokine pattern in sarcoidosis is expressed by bronchoalveolar CD4+ and CD8+ T cells. Clin Exp Immunol 2000; 122: 241-248.

8 Bergeron A, Bonay M, Kambouchner M, et al. Cytokine patterns in tuberculous and sarcoid granulomas; correlation with histopathologic features of the granulomatous response. J Immunol 1997; 159: 3034-3043.

9 Nishioka Y, Manabe K, Kishi J, et al. CXCL9 and 11 in patients with pulmonary sarcoidosis: a role of alveolar macrophages. Clin Exp Immunol 2007; 149: 317-326.

10 Agostini C, Cassatella M, Zambello R, et al. Involvement of the IP10 chemokine in sarcoid granulomatous reactions. J Immunol 1998; 161: 6413-6420.

11 Katchar K, Eklund A, Grunewald J. Expression of Th1 markers by lung accumulated $\mathrm{T}$ cells in pulmonary sarcoidosis. J Intern Med 2003; 254: 564-571.

12 Miotto D, Christodoulopoulos P, Olivenstein R, et al. Expression of IFN- $\gamma$-inducible protein; monocyte chemotactic proteins 1,3 , and 4; and eotaxin in $\mathrm{T}_{\mathrm{H}} 1$ - and $\mathrm{T}_{\mathrm{H}} 2$-mediated lung disease. J Allergy Clin Immunol 2001; 107: 664-670.

13 Capelli A, di Stefano A, Lusuards M, et al. Increased macrophage inflammatory protein- $1 \alpha$ and macrophage inflammatory protein$1 \beta$ levels in bronchoalveolar lavage fluid of patients affected by different stages of pulmonary sarcoidosis. Am J Respir Crit Care Med 2002; 165: 236-241.

14 Callejas-Rubio JL, Ortego-Centeno N, Lopez-Perez L, et al. Treatment of therapy-resistant sarcoidosis with adalimumab. Clin Rheumatol 2006; 25: 596-597.

15 Baughman RP, Drent M, Kavuru M, et al. Infliximab therapy in patients with chronic sarcoidosis and pulmonary involvement. Am J Respir Crit Care Med 2006; 174: 795-802.

16 Homma JY, Abe C, Chosa $\mathrm{H}$, et al. Bacteriological investigation on biopsy specimens from patients with sarcoidosis. Jpn J Exp Med 1978; 48: 251-255.

17 Ishige I, Usui $\mathrm{Y}$, Takemura $\mathrm{T}$, et al. Quantitative PCR of mycobacterial and propionibacterial DNA in lymph nodes of Japanese patients with sarcoidosis. Lancet 1999; 354: 120-123.

18 Eishi Y, Suga M, Ishige I, et al. Quantitative analysis of mycobacterial and propionibacterial DNA in lymph nodes of Japanese and European patients with sarcoidosis. J Clin Microbiol 2002; 40: 198-204.

19 Nishioka Y, Yano S, Fujiki F, et al. Combined therapy of multidrugresistant human lung cancer with anti-P-glycoprotein antibody and monocyte chemoattractant protein- 1 gene transduction: the possibility of immunological overcoming of multidrug resistance. Int J Cancer 1997; 71: 170-177.

20 Baba M, Nishimura O, Kanzaki N, et al. A small-molecule, nonpeptide CCR5 antagonist with highly potent and selective anti-HIV-1 activity. Proc Natl Acad Sci USA 1999; 96: 5698-5703. 
21 Gao P, Zhou X-Y, Yashiro-Ohtani Y, et al. The unique target specificity of a nonpeptide chemokine receptor antagonist: selective blockade of two Th1 chemokine receptors CCR5 and CXCR3. J Leukoc Biol 2002; 73: 273-280.

22 Nishioka Y, Hirao M, Robbins PD, et al. Induction of systemic and therapeutic antitumor immunity using intratumoral injection of dendritic cells genetically modified to constitutively express interleukin-12. Cancer Res 1999; 59: 4035-4041.

23 Aono Y, Nishioka Y, Inayama Y, et al. Imatinib as a novel antifibrotic agent in bleomycin-induced pulmonary fibrosis in mice. Am J Respir Crit Care Med 2005; 171: 1279-1285.

24 Kishuku M, Nishioka Y, Abe S, et al. Expression of soluble vascular endothelial growth factor receptor-1 in human monocyte-derived mature dendritic cells contributes to their antiangiogenic property. J Immunol 2009; 183: 8176-8185.

25 Hashida N, Ohguro N, Nakai K, et al. Microarray analysis of cytokine and chemokine gene expression after predonisolone treatment in murine experimental autoimmune uveoretinitis. Invest Ophthalmol Vis Sci 2005; 46: 4224-4234.

26 Nishiwaki T, Yoneyama H, Eishi Y, et al. Indigenous pulmonary Propionibacterium acnes primes the host in the development of sarcoid-like pulmonary granulomatosis in mice. Am J Pathol 2004; 165: 631-639.
27 Ichiyasu H, Suga M, Matsukawa A, et al. Functional roles of MCP-1 in Propionibacterium acnes-induced, T cell-mediated pulmonary granulomatosis in rabbits. J Leukoc Biol 1999; 65: 482-491.

28 McCaskill JG, Chason KD, Hua X, et al. Pulmonary immune responses to Propionibacterium acnes in C57BL/6 and BALB/c mice. Am J Repir Cell Mol Biol 2006; 35: 347-356.

29 Iyonaga K, McCarthy KM, Schneeberger EE. Dendritic cells and the regulation of a granulomatous immune response in the lung. Am J Respir Cell Med Biol 2002; 26: 671-679.

30 Katchar K, Eklund A, Grunewald J. Expression of Th1 markers by lung accumulated $\mathrm{T}$ cells in pulmonary sarcoidosis. J Intern Med 2003; 254: 564-571.

31 Hartl D, Krauss-Etschmann S, Koller B, et al. Infiltrated neutrophils acquire novel chemokine receptor expression and chemokine responsiveness in chronic inflammatory lung disease. J Immunol 2008; 181: 8053-8067.

32 Reichel CA, Khandoga A, Anders H-J, et al. Chemokine receptors Ccr1, Ccr2, and Ccr5 mediate neutrophil migration to postischemic tissue. J Leukoc Biol 2006; 79: 114-122.

33 Samokhin AO, Bühling F, Theissig F, et al. ApoE-deficient mice on cholate-containing high-fat diet reveal a pathology similar to lung sarcoidosis. Am J Pathol 2010; 176: 1148-1156. 